equality is much simpler than the estimate of the magnitude of loudness. It was most important that the response of the telephones used should have a linear relation with the amplitude of the disturbance especially at high values of the amplitude.

The authors have made measurements of the magnitudes of common noises on various scales. In what follows, we give them in loudness units. Calling zero the threshold of hearing, the ticking of a watch at three feet would be unity. In a quiet saloon motorcar it would be 10 . Ordinary conversation at three feet would be 20 , but if in a suburban steam train with the window open it would be 50. A loud motor horn at 100 feet was found to be 100 and two circular saws at three feet 160 .

The effect of placing the source inside a building is very pronounced. An 800 cycle tone placed in an enclosure had a loudness of 41 , whilst outside it was only $2 \cdot 4$. In making these measurements it is vitally important to take the background of noise into consideration. It is a matter of everyday experience that one sound can 'drown' another. A list of typical noise levels is given. For example, a busy main street in a certain city had a noise level of 22 . When trains were passing it rose to 53. On a weekday on the ground floor of an office in the street with the windows open, the noise level was 22 , but shutting the windows reduced it to 11 . On a Sunday morning with the windows open it was 0.6 and closed 0.2 . In a dining car in a train travelling at 60 miles per hour the level was about 50, but in a tunnel it rose to 82 .

When apparatus is installed near a main street in a busy city, we have to consider a background of between 20 and 50 . In this case a comparatively loud noise is scarcely noticed. On the other hand, when a residential hotel has to be considered, special precautions have to be taken. The screening effect produced by adjacent buildings is sometimes of assistance. At certain hours of the night the background may be so low as 1 unit and a much lower noise emission would have to be aimed at. If the problem is to be adequately dealt with in quiet residential districts, some form of enclosure must be used.

\title{
Permeability Tuning in Radio-Frequency Circuits
}

$\mathrm{V}$ ARIABLE condensers are now so commonly employed in radio receivers to tune circuits including a constant inductance that the use, some years ago, of variable inductances or variometers with fixed condensers is apt to be forgotten. For some purposes, however, the latter arrangement may have considerable advantages. A paper by W. J. Polydoroff ${ }^{1}$ refers to the advantages, particularly in the matter of selectivity, which result from tuning radio receiver circuits in such a manner that the ratio of the inductance to the resistance of the circuit remains constant. These desirable results may be conveniently accomplished by a new type of ferro-inductance. The coil itself is designed to have the desired performance at the highest frequency in the band to be covered. The effective inductance is then increased to tune to lower frequencies by introducing a magnetic core into the field of the coil. As the core is inserted into the coil, more lines of the magnetic field are intercepted by the core, and in effect, the average permeability of the medium surrounding the coil increases from unity, for air, to a certain maximum when the coil is entirely encased in the core: hence the term 'permeability tuning'.

The successful application of this principle to radio-frequency circuits depends upon the production of an iron-core material having an appreciable permeability at the working frequency, but free from the property of introducing undesirable resistance into the circuit. For many years, thinly laminated iron and stranded cores have been used for audio frequencies, while compressed iron dust cores have also come into use for frequencies up to about 50 kilocycles per second. Quite recently, considerable attention has been paid to the use of both iron and high permeability alloys for the construction of these dust cores, in order to obtain the necessary high permeability without the accompaniment of serious losses at radio frequencies.

In his paper, Polydoroff describes the use of pure iron reduced by hydrogen as a primary material for radio cores. While hysteresis losses are apparently vanishingly small at radio-frequencies, the eddycurrents are proportional to the square of the frequency and to the length of the circular path around each minute particle. The research described was directed at the broadcast frequency band, 550-1,500 kilocycles per second, and in this band the optimum grain size of the iron proved to be about 5 microns in diameter. This iron powder is mixed with a suitable insulating varnish and compressed in heated moulds of the desired shape, using pressures up to twenty-five tons per square inch. The resultant product has the appearance of solid iron, exhibits fair mechanical strength, and can be machined in the usual manner. The effective permeability obtained in such materials varies from about 5 to 12 according to the pressure employed in the moulding process.

The paper describes the use of this type of iron core in various types of radio receiver circuit. A good quality single layer solenoid of small dimensions is used as the inductance, and the core is made of two parts, an outer cylindrical shell and an inner plug, so as to enclose the coil in the position of maximum inductance. A semi-fixed condenser is attached to the end of each coil, and this is initially adjusted to give resonance at the highest frequency required. The cores are mounted on a common platform and inserted in their respective coils by a single tuning control. Provision is made to move each coil or each core separately in order to produce synchronisation at the middle of the range.

In receivers employing as many as six tuned circuits, no difficulty has been experienced in maintaining synchronism and constancy of the inductance to resistance ratio throughout the whole frequency band. The arrangement is equally applicable to the supersonic heterodyne and the straight radiofrequency amplifier types of receiver, and the advantages of the latter with the possibility of increased selectivity may give rise to interesting developments in the future.

1 "Ferro-Inductors and Permeability Tuning", Proc. Inst. Rad. Eng., May, 1933. 